\title{
When dipping toast into a cup of tea leads to a scientific investigation
}

\author{
Philippe Marmottant, Florian Orthion, and Salima Rafaï \\ CNRS, Univ. Grenoble Alpes, LIPhy, F-38000 Grenoble, France
}

(Received 11 August 2019; accepted 18 September 2019)

\begin{abstract}
This article recounts a scientific investigation that started from the observation of an ordinary everyday phenomenon. In a cup of tea, dipping buttered toast produces on the surface of the tea beautifully evolving lenses, which are pierced repeatedly by moving holes. To unravel this dynamic observation, we show here how we simplified the system into its basic ingredients, and how we excluded several hypotheses such as thermal convection. We reproduce the phenomenon with an inverse emulsion of surfactant-laden water droplets. Depending on the spreading of the lens, we also observe and explain the formation of an oil lacework and "swimming" lenses. (0) 2019 American Association of Physics Teachers.

https://doi.org/10.1119/10.0000025
\end{abstract}

\section{INTRODUCTION}

Everyday life is full of phenomena that involve spreading liquids, and it is common practice to cite industrial applications such as painting, printing, or coating. Spreading of a liquid on another liquid is dictated by the spreading coefficient ${ }^{1-3}$ which determines if it is energetically favorable to replace the initial interface by new interfaces. Depending on the sign of the spreading coefficient, the liquid will then either spread or remain as a condensed droplet.

Here, we focus on an astonishing phenomenon that can be observed at home at breakfast time. If you dip a slice of buttered toast into your hot tea (an outrageous habit in some countries), and look carefully at the surface of the water, you will see a rapidly spreading lens of melted butter. This indicates that spreading of molten butter is favourable. However, further inspection reveals many holes appearing in the patches of molten butter. These vacancies repeatedly grow and move within the oily lens, creating a beautiful ballet of holes, see Fig. 1 (and Movie 1 in Supplementary Materials). Looking more closely at a single butter lens, we see repeated nucleation and expulsion of such holes, see Fig. 2 (Movie 2 in Supplementary Materials). Note that expulsion of a hole results in the ejection of a tiny satellite droplet.

The purpose of this article is two-fold. First, our aim is to discover the mechanisms that give rise to the phenomenon. Second, we wish to illustrate how a physicist behaves when confronted with a new observation such as this. We believe that self-observation like this is a good opportunity for demonstrating the essence of the work of a physicist, and also a timely occasion to unravel the philosophy of scientific research, in this case with a daily-life phenomenon in mind. We hope that this approach might be helpful for public outreach interactions.

As physicists, our observation of natural phenomena is influenced by our knowledge of interfacial mechanisms that we might have encountered in other well-known situations. And here, in this mug we can imagine lots of mechanisms that might possibly trigger such a phenomenon. Likely mechanisms include thermal gradient effects and convection, or capillary phenomena.

Our aim is to show how to shed light on a seemingly multifactorial system. More specifically, we will be guided by the following steps:

- Reduction: Show how the complexity of the problem can be reduced by eliminating mechanisms that have no influence;
- Hypothesis: Formulate a hypothesis;

- Reproduction: Construct an experimental model system that reproduces the observations and confirms the hypothesis;

- Position in existing knowledge: Link this phenomenon to other related phenomena in the literature.

As a bonus, we will find that the experimental model can lead us to the discovery of new phenomenon, with different spreading regimes.

\section{REDUCING A COMPLEX "NATURAL" PHENOMENON TO A MODEL SYSTEM}

In this part, we detail the steps that we undertook to unravel the mechanism causing the hole formation. This section can be read as a detective story!

\section{A. Reduction: Necessary ingredients, investigation by trial and elimination}

As the initial system is complex in terms of materials and methods, we undertook a series of tests to simplify it, see Table I. This investigation was not done randomly. It was carried out by keeping in mind possible physical phenomena.

At the start, the observations of hole formation and migration were made with butter on hot tea (Test 1). Are tea particles important for nucleating holes? No, because holes appear when hot water alone is used (Test 2). Note that we tested black, regular and rooibos tea with the same results. Is there thermal convection that might cause bubbles to be trapped and form holes? No, because molten butter lenses deposited on cold water also give rise to hole formation (Test 3). Is the viscosity of water important for the formation and migration of holes? If a bath of glycerol is used instead of water, holes appear but they migrate more slowly (Test 4). Is the type of fat important in the formation of holes? No, lenses of vegetable oil also exhibit holes when deposited on pure cold water (Test 5). The exact composition of each of the ingredients is of course dependent on the exact type of butter or the type of oil we choose. However tests with several different brands were unable to reveal any difference. We tested molten butter ( $82 \%$ fat, clarified: i.e., taking only the liquid part), vegetable butter (60\% fat), and the following oils: olive oil, walnut oil, colza oil, sunflower, a mixture of colza, sunflower, and linseed oil (Equilence 4, Casino). All ingredients were purchased at the local grocery store. Only the number of holes differed slightly when the oils were interchanged. 


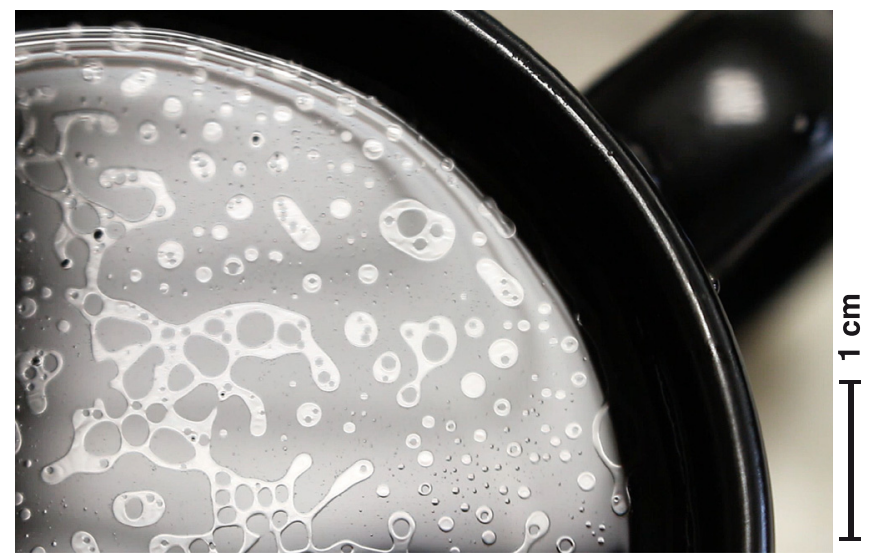

Fig. 1. Lenses of melted butter on hot water in a mug, showing rapidly moving hole structures. See also Supplementary Movie 1 (Ref. 19). The width of the field of view is here $5 \mathrm{~cm}$.

The key observation was the impact of filtering the liquid oil through a $0.2 \mu \mathrm{m}$ filter: no holes formed (Test 6$)$. This makes the presence of impurities in the butter the principal "suspect" in hole formation. But what are these impurities?

From this series of preliminary experiments, we can exclude the following phenomena: (i) liquid bath composition, since it works with pure water or glycerine, instead of tea (test 2 and 4), (ii) thermal convection effects, since it works with cold water (test 3), and (iii) fat composition effects, since it works with many different butters and oils (test 5).

\section{B. Hypothesis}

Our hypothesis is that impurities are present in butter, and that they contain surfactants. Butter is indeed an inverted emulsion that contains water in the form of tiny droplets ${ }^{4,5}$ stabilized by amphiphilic molecules that are naturally present in vegetable or animal oils.

The proposed mechanism is the following: these impurities fall very slowly inside the butter lens (Fig. 3(a)). Upon touching the lower surface, they release surfactants at the water-oil interface. Local deposition of surfactants induces what is called a Marangoni flow. Near the point of impact, the surfactant concentration is higher, which reduces the surface tension. A stress develops at the interface that drags

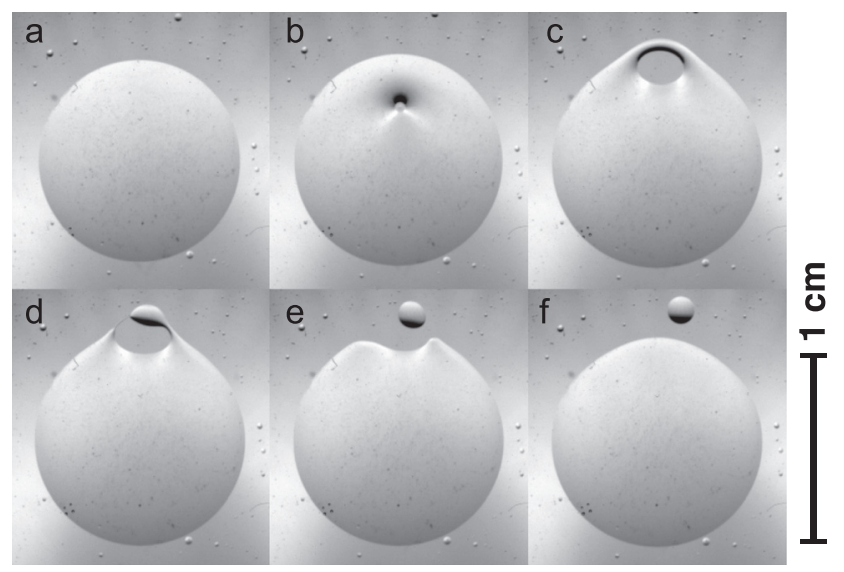

Fig. 2. Genesis of a hole in a molten butter lens: nucleation and migration on water at ambient temperature: (a) $\mathrm{t}=0 \mathrm{~s}$, (b) $\mathrm{t}=0.11 \mathrm{~s}$, (c) $\mathrm{t}=0.28 \mathrm{~s}$, (d) $\mathrm{t}=0.33 \mathrm{~s}$, (e) $\mathrm{t}=0.34 \mathrm{~s}$, and (f) $\mathrm{t}=0.41 \mathrm{~s}$. See also Supplementary Movie 2 . The size of the initial lens is $1 \mathrm{~cm}$.
Table I. Tests performed in seeking the conditions for the presence of holes.

\begin{tabular}{lccc}
\hline \hline Test \# & Liquid in the lens & Bath & Result \\
\hline 1 & Molten butter & Hot tea & Holes \\
2 & Molten butter & Hot water & Holes \\
3 & Molten butter & Cold water & Holes \\
4 & Molten butter & Glycerin & Holes, migration slower \\
5 & Vegetable Oil & Cold water & Holes \\
6 & Filtered vegetable Oil & Cold water & No holes \\
\hline \hline
\end{tabular}

liquid in the direction of higher surface tension, i.e., away from the point of impact (see red lines of Fig. 3(b)). The films can become so thin that a hole opens, as described in a study where surfactant molecules were injected at the bottom of an oil layer. ${ }^{6}$ The hole then migrates to the edge in order to minimise its surface (see Fig. 3(c)). Thus, closer to the edge, the hole depth becomes smaller.

\section{Experimental model}

On the basis of this hypothesis, we produced a liquid with the smallest possible set of well controlled ingredients: (i) filtered oil, in order to remove impurities, and (ii) a solution of sodium dodecyl sulfate (SDS) in water. Vigorous shaking of this liquid created an emulsion composed of tiny submillimetre sized droplets of SDS solution. As the density of droplets is greater than that of oil, they accordingly fall slowly towards the bottom interface of the lens. The material we used was a vegetable oil (Equilence 4, containing colza, sunflower, and linseed) filtered through a $0.2 \mu \mathrm{m}$ filter. Its surface tension, measured by the drop weight method, ${ }^{7}$ is $27.5 \mathrm{mN} / \mathrm{m}$.

When deposited on water our experimental model system closely reproduces the features observed earlier, with nucleation of holes at arbitrary positions and their migration towards the edge of the lens, see Fig. 4. At this point, we declare that the hypothesis is validated!

\section{State of the art of surfactant and Marangoni induced phenomena with floating liquid lenses}

Once we have elucidated the principal ingredients underlying the natural phenomenon, we now want to place this new result in the context of other studies where dynamic effects are observed on floating liquid lenses. The Marangoni flow where a water bump is created on the lower surface and then a hole was described in Refs. 6, 8, and 9. Although that study investigated somewhat thicker layers of oil $(3-13 \mathrm{~mm})$ covering all the liquid bath, and the holes were initiated by injecting $20 \mu \mathrm{l}$

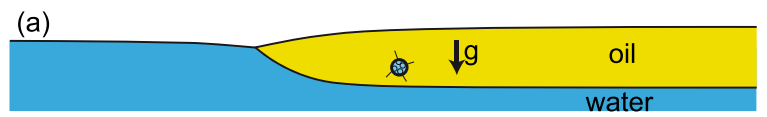

(b)

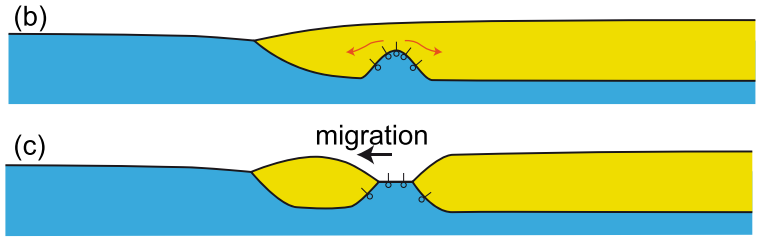

Fig. 3. Our hypothesis: (a) impurities containing surfactants fall under gravity until they touch the bottom surface, (b) liberating surfactants at the lower interface, which creates a strong Marangoni flow that pushes liquid away, and (c) a hole opens and migrates towards the edge of the lens. 

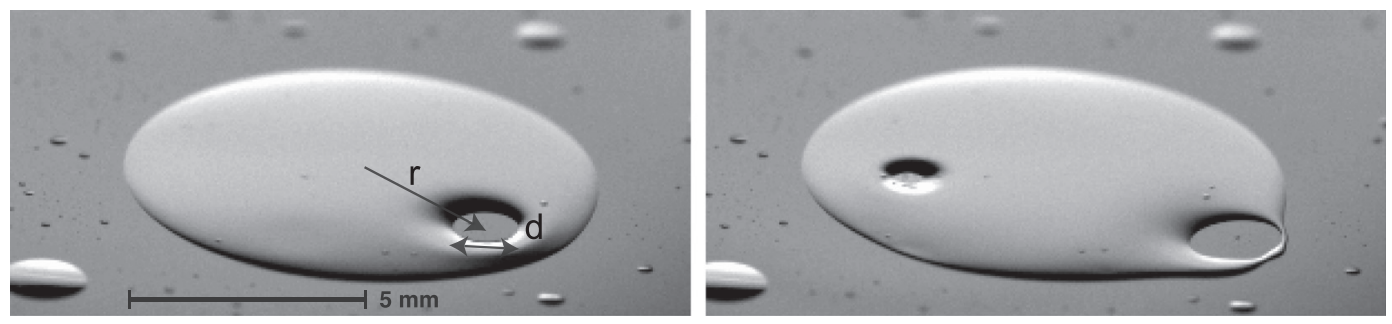

Fig. 4. Side view of a hole migrating in a reconstituted model system: emulsion (1\% in volume) of water/SDS droplets in filtered vegetable oil. Time between images: $1.5 \mathrm{~s}$. Lens diameter $10 \mathrm{~mm}$. The emulsion contains water droplets with SDS concentration $c=8 \times 10^{-2}$ mol/l, which is 10 times greater than the critical micellar concentration (CMC). See also Supplementary Movie 3.

droplets, the fundamental mechanism is similar to that in the present study. In this work, the model system droplets are of sub-millimetre size.

Numerous other impressive dynamic effects on floating lenses containing surfactants have been reported in the literature. Oil lenses containing surfactant can produce repeated oscillations of their radius. ${ }^{10}$ This effect was attributed to evaporation of the surfactants as they spread at the water-air interface. Droplets containing a mixture of a volatile liquid and surfactants give rise to spectacular effects, such as spreading and bursting into myriads of small droplets, ${ }^{11}$ or large amplitude oscillations. ${ }^{12}$

\section{CLOSER INSPECTION WITH A HIGH-SPEED CAMERA}

\section{A. Fate of a hole within a lens}

We now focus on the fate of each hole after its nucleation. For this purpose, we imaged a lens using a high speed camera (Miro 4, Vision Research) in reflexion mode, see Fig. 4. The images were analysed to determine the hole diameter $d$ and its distance from the centre of the lens $r$, see Fig. 5. We observed the following steps in hole formation:
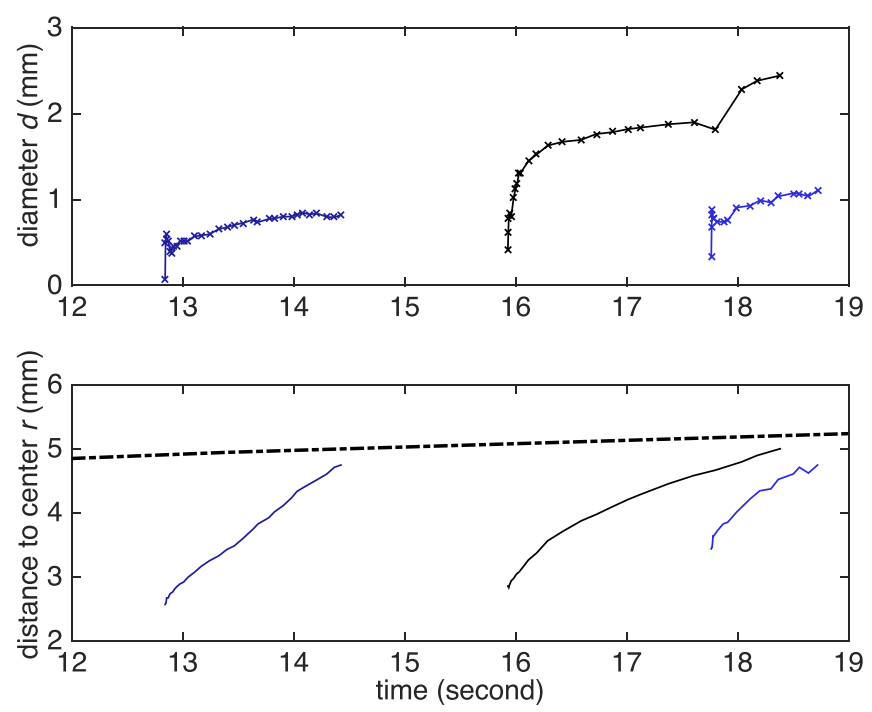

Fig. 5. Measurements from images of the set-up depicted in Fig. 4. The curve showing the hole diameter of several holes as a function of time shows a fast initial expansion (top figure). The migration of the holes away from the center of the lens is well captured by the curve showing the distance of the hole centre to the lens centre (bottom). The dotted-dashed line represents the position of the lens edge. Time $t=0 \mathrm{~s}$ corresponds to the deposition of the lens on water.
(1) Nucleation: A fast expansion phase typically lasting between 0.01 and $0.02 \mathrm{~s}$ (see Fig. 6), with a growth velocity that can be as high as $100 \mathrm{~mm} / \mathrm{s}$. Note that the nucleation is quite energetic, since it induces a circular capillary wave starting from the hole and travelling over the liquid bath.

(2) Migration: With a velocity that is roughly constant at about $1 \mathrm{~mm} / \mathrm{s}$, slowing down near the edge of the lens, see Fig. 5 , bottom.

(3) Ejection. When the hole arrives near the edge, a ridge forms and drains/pinches off. It can produce a satellite droplet when the ridge, looking like a "handle" (Fig. 2(c)) pinches off at the two points of attachment to the main lens where holes are ejected.

There is a large variability in hole size and sometimes we see only a small bump emitting a capillary wave but no hole. According to LeRoux, ${ }^{9}$ the final size of each hole is an increasing function of the number of surfactant molecules. In fact, the larger the amount of surfactant, the larger are the Marangoni stresses and the longer their duration. More specifically, the final size of the holes depends on the amount of insoluble surfactant. Here the variability in the size of the holes may then be explained by the variation of the size of the droplets/impurities touching the interface. Droplets/impurities that are too small would merely give rise to a bump.

\section{B. Very large lens: Inflating lace}

Another regime appears if a knob of solid butter is dropped directly on top of hot water, causing an extended

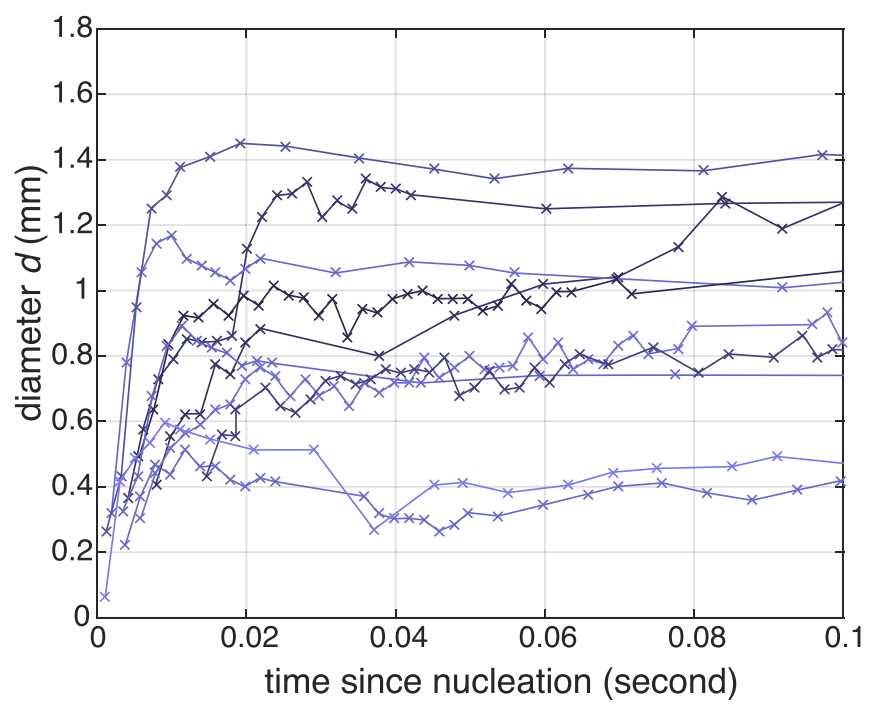

Fig. 6. Hole diameter as a function of the time after nucleation. The nucleation time is found by extrapolation of the curves of Fig. 5 (top). 
film of melted butter to expand rapidly over the whole surface of the container. A beautiful changing lace structure appears: Holes expand all over the film, participating in the film growth, see Fig. 7 and Supplementary Movie 4. Note there is no clear migration.

Finally, the bridges between holes break, causing the holes to coalesce and form isolated lenses, see Fig. 7.

The phenomenon can be reproduced using the same model system as for the hole formation. Deposition of a large amount of emulsion (water in oil) on water creates a thin film with growing holes, see Fig. 8. The amount is so large that the film thickness is nearly uniform, and migration of holes does not occur.

\section{Swimming lens}

Finally, we wish to share a remarkable observation on a lens of molten butter that did not spread as a film but remained concentrated as a droplet. The edge of the droplet changes constantly with time, with indentations appearing and disappearing, see Fig. 9. The droplet moves erratically, reminiscent of self-propelled objects, with a velocity of about $1 \mathrm{~mm} / \mathrm{s}$.

Marangoni stresses have been shown to induce selfpropulsion in many kinds of systems: oil droplets on water, ${ }^{13}$ immersed droplets of water in oil-surfactant mixtures, ${ }^{14}$ or heated spheres at a water-air interface. ${ }^{15}$ Marangoni induced self-propulsion is also responsible for a phenomenon mentioned for the first time in 1860 by scientists who were contemporaries of the Italian physicist Carlo Marangoni, namely, the motion of camphor on the surface of water. ${ }^{16}$ This phenomenon has been generalised to what are called camphor boats, which have been extensively studied. ${ }^{17,18}$

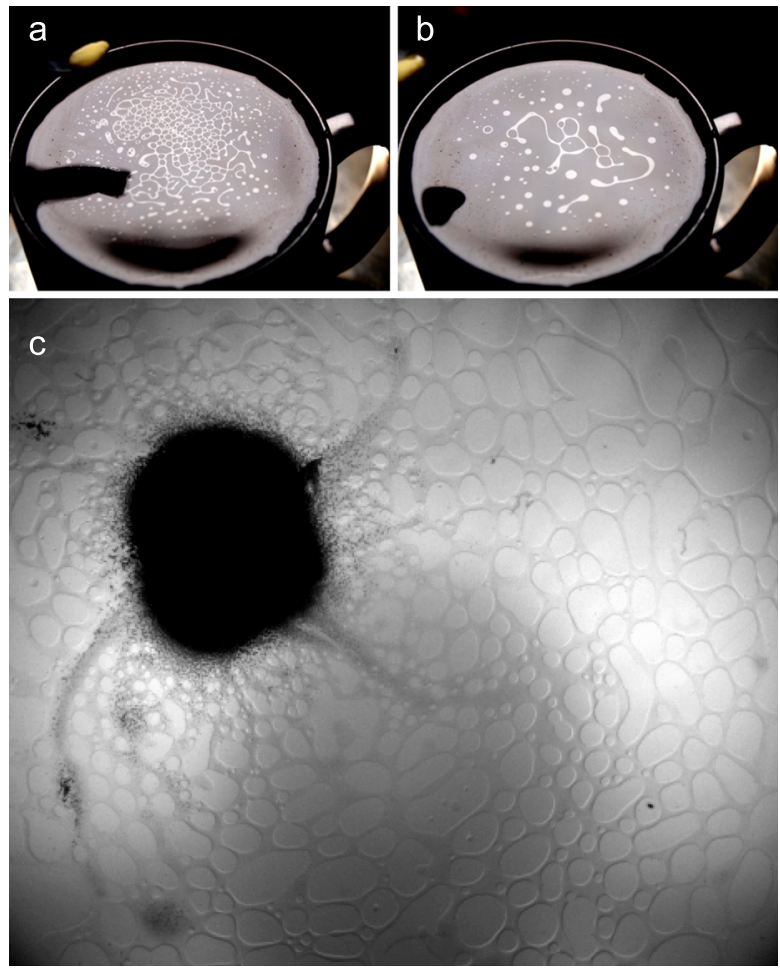

Fig. 7. Butter melting on hot water: formation of an evolving "lace," with growing holes (top pictures). The lace starts from the knob of butter (bottom picture). The diameter of the knob of butter is $6 \mathrm{~mm}$. See also Supplementary Movie 4.

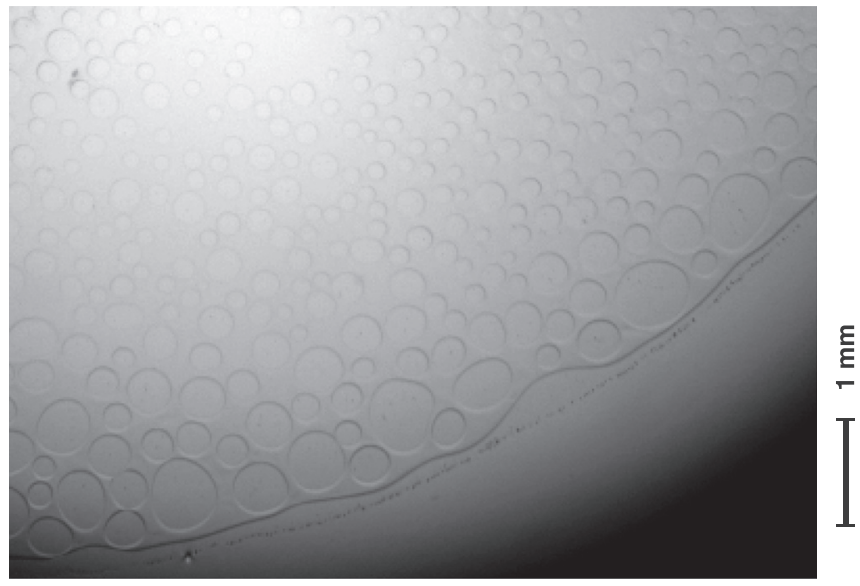

Fig. 8. Reconstitution of a lace pattern, using an emulsion of water/SDS droplets in vegetable oil spreading on pure water. The width of the field of view is $7.4 \mathrm{~mm}$.

\section{DISCUSSION: TOWARDS A CONSISTENT PICTURE COVERING SEVERAL PHENOMENA}

In Sec. III, we saw that our initial interrogation about an everyday-life phenomenon gave rise to unexpectedly many more results. Our working hypothesis was validated experimentally, which encouraged us to develop the measurements further and to observe new phenomena. Here, we try to rationalise these into a coherent picture.

We observed a variety of phenomena: migrating holes in oily lenses, lacework-like patterns of the fatty phase and even swimming-like motion of the lenses. These different regimes might be related to the ability of the oil to spread on water. Visual inspection seems to qualitatively show that

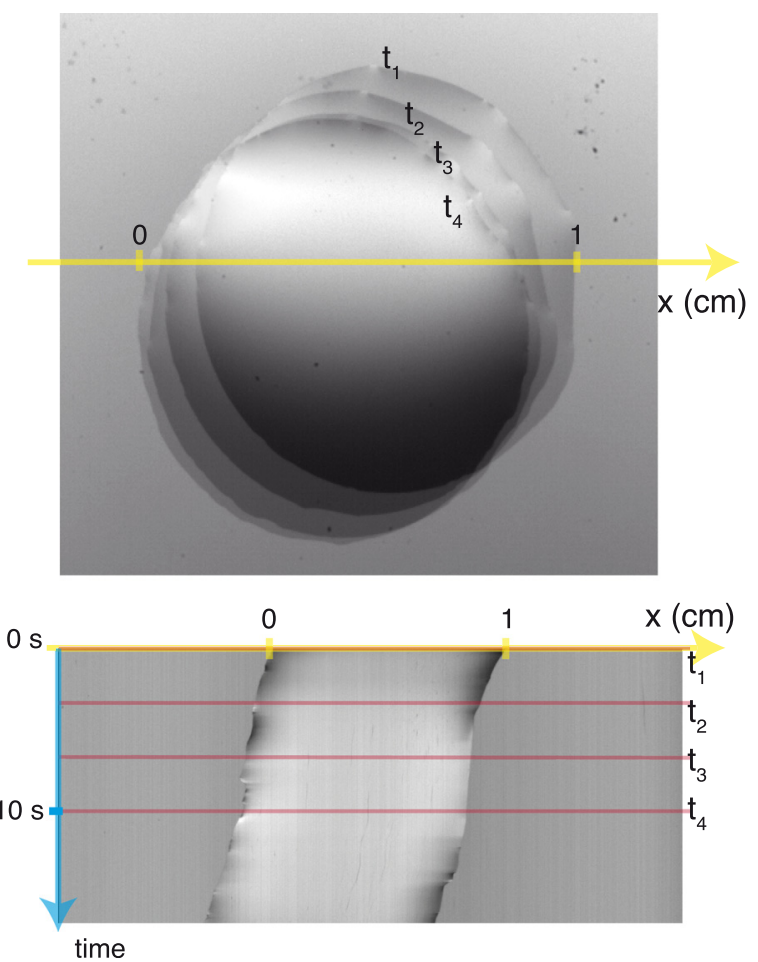

Fig. 9. Swimming lens at four different times (top) and spatio-temporal diagram obtained by the superposition of the lines at different times (bottom). The lens is around $10 \mathrm{~mm}$ in diameter. See also Supplementary Movie 5. 
when the oily phase spread totally on water, we rather end up with the lace-like pattern. In this case, the formation of holes and the spreading occur simultaneously so that the oil film thickness seems homogeneous. In the case of intermediate spreading, we observe the formation of migrating holes. Finally, when the oil droplet is too thick, holes do not develop except at the very edge. In this case, we could observe undulations of the droplet boundary and the whole droplet is then propelled erratically. Quantitative measurements would be needed to consolidate these interpretations but those lie beyond the scope of this manuscript.

\section{PERSPECTIVES}

We hope we have succeeded in showing how a curiositydriven approach to an everyday phenomenon can lead to observations that are interesting and unexpectedly varied. We propose this example as a teaching support to explain the scientific reasoning and the background knowledge that are necessary to formulate a hypothesis. The experiment is a beautiful example of Marangoni interfacial phenomena, to which belong other well-known demonstration experiments, such as what happens when soap is dipped into the surface of water previously covered for instance with pepper particles.

\section{ACKNOWLEDGMENTS}

The authors thank Thomas Combriat for fruitful discussions and Erik Geissler for carefully reading the manuscript. P.M. and S.R. would like to acknowledge Yves Couder for sharing his inspiring way of doing physics.

${ }^{1}$ L. Leger and J. F. Joanny, "Liquid spreading," Rep. Prog. Phys. 55, 431-486 (1992).

${ }^{2}$ Daniel Bonn, Jens Eggers, Joseph Indekeu, Jacques Meunier, and Etienne Rolley, "Wetting and spreading," Rev. Mod. Phys. 81, 739-805 (2009).

${ }^{3}$ Jacco H. Snoeijer and Bruno Andreotti, "Moving contact lines: Scales, regimes, and dynamical transitions," Ann. Rev. Fluid Mech. 45, 269-292 (2013).
${ }^{4}$ G. Bobe, S. Zimmerman, E. G. Hammond, A. E. Freeman, P. A. Porter, C. M. Luhman, and D. C. Beitz, "Butter composition and texture from cows with different milk fatty acid compositions fed fish oil or roasted soybeans," J. Dairy Sci. 90, 2596- 2603 (2007).

${ }^{5}$ A. C. Juriaanse and I. Heertje, "Microstructure of shortenings, margarine and butter-a review," Food Struct. 7, 181-188 (1988), see https:// digitalcommons.usu.edu/foodmicrostructure/vol7/iss2/8.

${ }^{6}$ Rodrigo Leite Pinto, Sébastien Le Roux, Isabelle Cantat, and Arnaud SaintJalmes, "Enhanced interfacial deformation in a Marangoni flow: A measure of the dynamical surface tension," Phys. Rev. Fluids 3, 024003 (2018).

${ }^{7}$ M. C. Wilkinson, "Extended use of, and comments on, the drop-weight (drop-volume) technique for the determination of surface and interfacial tensions," J. Colloid Interface Sci. 40, 14-26 (1972).

${ }^{8}$ Matthieu Roché, Zhenzhen Li, Ian M. Griffiths, Sébastien Le Roux, Isabelle Cantat, Arnaud Saint-Jalmes, and Howard A. Stone, "Marangoni flow of soluble amphiphiles," Phys. Rev. Lett. 112, 208302 (2014).

${ }^{9}$ Sébastien Le Roux, "Marangoni effect at fluid interfaces," Ph.D. thesis, Université Rennes 1 (2015).

${ }^{10}$ Roman Stocker and John W. M. Bush, "Spontaneous oscillations of a sessile lens," J. Fluid Mech. 583, 465-475 (2007).

${ }^{11}$ L. Keiser, H. Bense, P. Colinet, J. Bico, and E. Reyssat, "Marangoni bursting: Evaporation-induced emulsification of binary mixtures on a liquid layer," Phys. Rev. Lett. 118, 074504 (2017).

${ }^{12}$ F. Wodlei, J. Sebilleau, J. Magnaudet, and V. Pimienta, "Marangonidriven flower-like patterning of an evaporating drop spreading on a liquid substrate," Nat. Commun. 9, 820 (2018).

${ }^{13}$ Yong-Jun Chen, Yuko Nagamine, and Kenichi Yoshikawa, "Self-propelled motion of a droplet induced by Marangoni-driven spreading," Phys. Rev. E 80, 016303 (2009).

${ }^{14}$ Ziane Izri, Marjolein N. van der Linden, Sébastien Michelin, and Olivier Dauchot, "Self-propulsion of pure water droplets by spontaneous Marangoni-stress-driven motion," Phys. Rev. Lett. 113, 248302 (2014).

${ }^{15}$ A. Girot, N. Danné, A. Würger, T. Bickel, F. Ren, J. C. Loudet, and B. Pouligny, "Motion of optically heated spheres at the water-air interface," Langmuir 32, 2687-2697 (2016).

${ }^{16}$ Charles Tomlinson and Allen Miller, "On the motions of camphor on the surface of water," Proc. R. Soc. London 11, 575-577 (1862).

${ }^{17}$ Satoshi Nakata, Masaharu Nagayama, Hiroyuki Kitahata, Nobuhiko J. Suematsu, and Takeshi Hasegawa, "Physicochemical design and analysis of self-propelled objects that are characteristically sensitive to environments," Phys. Chem. Chem. Phys. 17, 10326-10338 (2015).

${ }^{18}$ Ronan Kervil, "Active matter and flows: Bacterial jets and interfacial swimmers," Ph.D. thesis, Université de Lyon (2018).

${ }^{19}$ See Supplementary Material at https://doi.org/10.1119/10.0000025 for Supplementary Movies 1-5. 